\title{
Efetividade de mantedores de espaço em odontopediatria: revisão sistemática
}

\section{Effectiveness of space maintainers in pediatric dentistry: a systematic review}

\author{
Aryane Marques Menegaz* \\ Morgana Favetti* \\ Douver Michelon ${ }^{* * *}$ \\ Marina Sousa Azevedo** \\ Catiara Terra da Costa**
}

\section{Resumo}

Objetivo: o objetivo deste estudo é avaliar por meio de revisão sistemática a efetividade dos dispositivos na manutenção do espaço para dentes permanentes quando da perda precoce do dente decíduo. Materiais e método: realizou-se uma pesquisa bibliográfica de estudos publicados até março de 2014, principalmente nas bases de dados PubMed e SciELO. Os critérios para seleção foram: estudos em ensaios clínicos randomizados (ECR) e ensaios clínicos controlados (ECC), com crianças de seis a doze anos com dentição decídua ou mista, com perda precoce de dentes decíduos na região anterior e/ou posterior. Foram considerados todos os tipos de mantedores de espaço tanto removíveis quanto fixos. Os desfechos considerados foram avaliação clínica, radiográfica e/ou análise de modelos. A análise dos textos e a coleta de dados foram realizadas por dois revisores de forma independente. Resultados: a busca resultou em um total de 438 artigos, após a remoção das duplicatas, e apenas um contemplou todos os critérios de inclusão. $O$ artigo que preencheu os critérios comparou dois diâmetros de fio ortodôntico utilizados para a confecção do aparelho mantedor de espaço, arco lingual, referindo que o mais efetivo foi confeccionado com fio de menor calibre $(0,9 \mathrm{~mm})$ quando comparado ao de maior calibre $(1,25 \mathrm{~mm})$. Essa diferença ocorreu devido às falhas que ocorrem com maior frequência no dispositivo de maior calibre. Considerações finais: evidenciou-se, por meio deste estudo, que devido à carência de ensaios clínicos, não é possível definir o mantedor de espaço mais efetivo, o que torna necessário o desenvolvimento de novos estudos com delineamentos adequados para responder essa questão de forma precisa.

Palavras-chave: Odontopediatria. Revisão sistemática. Mantenedor de espaço. Ortodontia. Crianças.

\section{Introdução}

Os dentes decíduos, além de importantes para o bom desempenho da função mastigatória, para articulação, fonação e oclusão estética da criança, são os melhores mantedores de espaço proximal e funcional. Isso devido à presença da coroa clínica, do periodonto e das raízes que guiam a irrupção dos dentes sucessores permanentes, permitindo o desenvolvimento adequado da dentição humana ${ }^{1,2}$.

A perda precoce dos dentes decíduos, especialmente dos molares, pode determinar sérias alterações no desenvolvimento da oclusão da dentição decídua, mista e, consequentemente, da dentição permanente. A perda parcial ou total da estrutura dentária acarreta uma diminuição do espaço disponível

Graduandas do curso de Odontologia da Universidade Federal de Pelotas, Pelotas, Rio Grande do Sul, Brasil

- Professoras doutoras em Odontopediatria, Faculdade de Odontologia, Universidade Federal de Pelotas, Pelotas, Rio Grande do Sul, Brasil

*** Professor doutor em Ortodontia, Faculdade de Odontologia Universidade Federal de Pelotas, Pelotas, Rio Grande do Sul, Brasil 
no arco dentário provocando um desequilíbrio estrutural e funcional ${ }^{3-5}$. A perda de um dente decíduo é considerada precoce ou prematura quando ocorre antes do tempo de sua esfoliação normal, quando um dente decíduo é perdido antes do sucessor permanente ter começado sua erupção (formação coronária completa e formação radicular já iniciada) ${ }^{6}$.

A principal causa da perda precoce de molares decíduos é a cárie, e no Brasil a prevalência é alta. Isso se deve aos problemas socioeconômicos aliados ao falso conceito popular de que os dentes decíduos não são importantes, pois serão substituídos por dentes permanentes. Sendo assim, o tratamento adequado para os dentes decíduos pode ficar negligenciado $0^{4,6-10}$.

Inclinação ou migração dos dentes adjacentes para o espaço do dente perdido, com consequente diminuição do perímetro do arco dentário, extrusão dos dentes antagonistas, desvio da linha média, apinhamento, impactação dentária e instalação de hábitos bucais nocivos, são as consequências mais frequentes encontradas nos pacientes que perderam precocemente dentes decíduos, e isso favorece a instalação de má oclusão na dentição permanente ${ }^{6,11}$.

Quando todos os tratamentos possíveis são realizados, e a exodontia do dente decíduo for a melhor indicação, mesmo que precocemente em relação à época ideal de desenvolvimento da dentição, um mantedor de espaço é indicado. Apesar da grande variedade de engenhosos mantedores, pode-se utilizar um dispositivo bastante simples, pois sua função é preservar o espaço para que o sucessor permanente possa irromper adequadamente ${ }^{6,12}$, mas 0 tipo a ser usado vai depender de cada caso individualmente.

Diante da perda dentária precoce, e no intuito de evitar o estabelecimento da má oclusão, a literatura recomenda o uso de mantedores de espaço. Sendo assim, este trabalho visa revisar sistematicamente a efetividade dos mantedores de espaço utilizados quando da perda precoce de dentes decíduos em crianças de seis a doze anos, por meio da busca de ensaios clínicos controlados e/ou randomizados.

\section{Materiais e método}

Este estudo caracteriza-se como uma revisão sistemática da literatura com o objetivo de avaliar a efetividade dos mantedores de espaço mais utilizados nos pacientes infantis quando há perda precoce de dentes decíduos.

\section{Critérios de inclusão dos estudos selecionados}

Foram selecionados para este estudo ensaios clínicos randomizados (ECRs) e ensaios clínicos controlados (ECCs) que respondessem a questão: os mantedores de espaço são efetivos para manter o diâmetro do arco em crianças de seis a doze anos que perderam precocemente os dentes decíduos?

\section{Tipos de participantes}

Crianças de seis a doze anos de idade com dentição mista que tivessem perdido precocemente pelo menos um dente decíduo na região anterior e/ou posterior por qualquer motivo.

\section{Tipos de intervenções}

Utilização de mantedores de espaço quando da perda precoce de dentes decíduos tanto na região anterior quanto posterior. Foram considerados todos os tipos de mantedores de espaço, tanto removíveis quanto fixos. Recuperadores de espaço e aparelho de irrupção guiada não foram considerados para esta revisão.

\section{Tipos de desfechos}

Manutenção do espaço para a irrupção de dentes permanentes anteriores e/ou posteriores, verificada por meio de avaliação clínica, radiográfica e/ou análise de modelos.

\section{Estratégia de busca dos artigos}

A estratégia de busca dos ensaios envolveu uma pesquisa nas bases de dados: PubMed Central e Scielo, utilizando combinações dos termos presentes no Medical Subject Headings e seus correspondentes no Descritores em Ciências da Saúde: child, children, space maintainer, space maintenance, space maintaining, space maint*, orthodontic, space maintenance and orthodontic, space maintainer and orthodontic", "space maintainer or orthodontic" (Tabela 1) . Não foram usados limites para data de publicação nem idioma, as buscas foram realizadas em março de 2014 e todos os estudos disponíveis até essa data foram considerados.

As referências dos artigos selecionados foram checadas para buscar estudos adicionais que eventualmente não tivessem sido encontrados nas buscas. 
Tabela 1 - Bases de dados, estratégia de busca e número de artigos

\begin{tabular}{|c|c|c|}
\hline $\begin{array}{c}\text { Bases de dados } \\
\text { eletrônicos utilizados } \\
\text { pesquisa }\end{array}$ & $\begin{array}{l}\text { Estratégia de busca } \\
\text { (palavras-chaves) }\end{array}$ & $\begin{array}{l}\text { Número } \\
\text { de artigos } \\
\text { recuperados }\end{array}$ \\
\hline PubMed & $\begin{array}{l}(((((((\text { Children[Text Word] }) \text { OR Child[MeSH Terms])) AND (((orthodontics[MeSH Terms]) } \\
\text { OR orthodontics, preventive[MeSH Terms]) OR orthodontics[Text Word]) OR preventive } \\
\text { orthodontics[MeSH Terms])) AND ((space maintenance[MeSH Terms]) OR space maint*[Text } \\
\text { Word])))) AND ((randomized controlled trial[pt] OR controlled clinical trial[pt] OR randomized } \\
\text { controlled trials[mh] OR random allocation[mh] OR double-blind method[mh] OR single-blind } \\
\text { method[mh] OR clinical trial[pt] OR clinical trials[mh] OR ("clinical trial"[tw]) OR ((singl*[tw] OR } \\
\text { doubl*[tw] OR trebl*[tw] OR tripl*[tw]) AND (mask*[tw] OR blind*[tw])) OR ("latin square"[tw]) } \\
\text { OR placebos[mh] OR placebo*[tw] OR random*[tw] OR research design[mh:noexp] OR follow- } \\
\text { up studies[mh] OR prospective studies[mh] OR cross-Over studies[mh] OR control*[tw] OR } \\
\text { prospectiv*[tw] OR volunteer*[tw]) NOT (animal[mh] NOT human[mh]))) }\end{array}$ & 116 \\
\hline SciELO & $\begin{array}{l}\text { tw:((tw:("ortodontia/mt")) OR (tw:(ortodontia)) AND (tw:("mantenedor de espaco/mt")) OR } \\
\text { (tw:(mantendedor)) ) AND (instance:"regional") AND ( type_of_study:("clinical_trials")) }\end{array}$ & 322 \\
\hline
\end{tabular}

\section{Análise e coleta de dados}

Dois pesquisadores (A. M. M e M. F.) avaliaram os artigos de forma independente, selecionando aqueles que correspondiam aos critérios de inclusão. Desacordos foram resolvidos após a apreciação por um terceiro avaliador (C. T. C). Após a seleção, os dados coletados foram: tipo de estudo, local, critérios de seleção, número e idade dos participantes, tipo de mantedores usados, forma de coleta do desfecho e conclusão/resultados.

\section{Resultados}

As buscas nas bases de dados PubMed e SciELO resultaram em um total de 438 artigos, e após a remoção de duplicata, restaram 437 artigos. Os avaliadores iniciaram então a análise dos títulos e resumos. Após essa etapa, um total de 427 artigos foi excluído. Restaram dez artigos, que foram verificados na íntegra (Figura 1). Desses, apenas um contemplou todos os critérios de inclusão: Effectiveness of a lower lingual arch as a space holding device ${ }^{13}$.

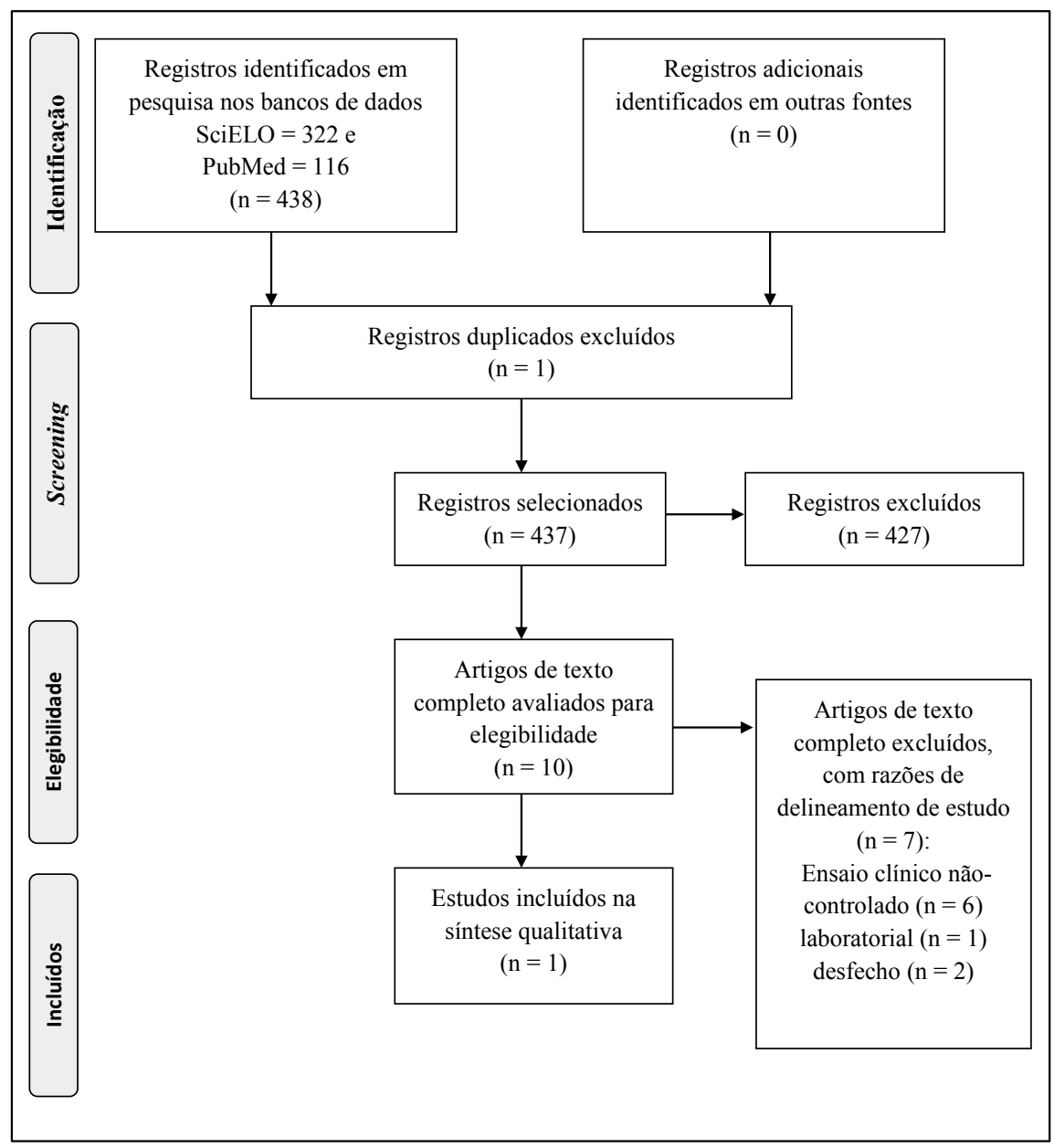

Figura 1 - Fluxograma da seleção dos estudos - Adaptado de Prisma statement ${ }^{14}$ 
Dos nove artigos excluídos, sete não correspondiam ao delineamento de estudo procurado ${ }^{15-21}$. Outros dois artigos foram excluídos ${ }^{22,23}$, apesar de serem incluídos nos critérios de tipo de estudo e faixa etária, por não possuírem o desfecho procurado. Um dos trabalhos avaliou os efeitos do tratamento ortodôntico na dentição mista com um aparelho de erupção guiada ${ }^{22}$, e o outro referiu que o uso de mantedor de espaço removível limitou o incremento de crescimento na região de caninos ${ }^{23}$.

\section{Características do artigo selecionado}

$\mathrm{O}$ artigo Effectiveness of a lower lingual arch as a space holding device ${ }^{13}$ foi produzido em Irbid, Jordânia, e publicado no ano de 2010. É um ensaio clínico controlado randomizado que avaliou a efetividade do arco lingual (LLHA - lower lingual holding arch) na manutenção do comprimento do arco frente à perda do segundo molar decíduo inferior. A comparação foi realizada com a confecção dos aparelhos com fio de aço inoxidável calibres $0,9 \mathrm{~mm}$ e 1,25 $\mathrm{mm}$.

A amostra estudada foi composta por 44 indivíduos que procuraram atendimento na clínica de ortodontia da Universidade da Jordânia, numerados aleatoriamente e divididos em números pares para o grupo 1 e ímpares para o grupo 2. Os pacientes do grupo 1 utilizaram o arco lingual confeccionado com fio de menor calibre, e os do grupo 2 com o de maior calibre. O grupo 3, descrito como grupo controle e que não recebeu nenhum tratamento, era composto por indivíduos que estavam em tratamento no centro de odontologia da Universidade da Jordânia. O grupo 1 foi composto por vinte indivíduos, doze do sexo masculino e oito do sexo feminino, com idade média de 10,76 anos; o grupo 2, por 24 indivíduos, doze de cada sexo, com idade média 10,57 anos; e o grupo 3 (controle), composto por 23 indivíduos, quinze do sexo masculino e oito do sexo feminino, com idade média de 10,63 anos. Os critérios para a seleção da amostra do estudo foram: dentição mista, classe I ou II, apinhamento menor que $2 \mathrm{~mm}$, sobremordida normal ou aumentada, um ou ambos segundos molares decíduos perdidos ou indicados para extração e sem ausência congênita.

$\mathrm{O}$ artigo utilizou telerradiografias de perfil, radiografias panorâmicas e análise dos modelos de estudo para avaliar a efetividade do mantedor de espaço, e os eventuais problemas que pudessem ocorrer durante o decorrer do estudo foram resolvidos em até 24 horas para não causar danos aos resultados. A pesquisa resultou que os LLHAs confeccionados com fio ortodôntico de calibre $0,9 \mathrm{~mm}$ foram superiores em termos de preservação do comprimento do arco.

\section{Discussão}

As perdas precoces de dentes decíduos têm sido frequentemente estudadas devido à sua relevância e associação com o surgimento de anormalidade de oclusão ${ }^{24}$, para isso, na fase da dentição decídua ou mista, os mantedores de espaço são utilizados para manter o espaço correspondente ao dente sucessor permanente. A perda do comprimento do arco resultante desse processo pode levar ao desenvolvimento de várias má oclusões na dentição permanente, justificando estudos pelo esclarecimento da efetividade dos mantedores conhecidos ${ }^{6,11}$

Os mantedores de espaço utilizados em odontopediatria podem ser fixos ou removíveis, funcionais ou não, e podem reabilitar a região anterior e/ou posterior. Os mais conhecidos são placas de acrílico com grampos ortodônticos, que servem tanto para a região anterior quanto posterior, sendo utilizados também para a recuperação da estética; arco lingual, botão palatino, banda-alça e dispositivos confeccionados com fio ortodôntico e resina fotopolimerizável são dispositivos fixos e comumente aplicados à região posterior ${ }^{6,25}$

Banda-alça é geralmente o aparelho de escolha quando ocorre a perda prematura unilateral dos primeiros ou segundos molares decíduos superiores ou inferiores. Com a perda do segundo molar decíduo, o aparelho de Nance ou a barra transpalatina podem ser utilizados no arco superior. No arco inferior, opta-se pelo arco lingual (LLHA). O mantedor de espaço ideal deve ser simples, resistente e de fácil higienização. Não deve restringir o crescimento normal ou interferir na oclusão, fala e mastigação ${ }^{26}$

Alguns estudos tentam comparar diferentes tipos de mantedores com o propósito de verificar qual apresenta melhor capacidade de manutenção dos espaços, mas os resultados não apresentam diferenças estatisticamente significativas. Assim como tentam verificar outros desenhos de mantedores que apresentem a mesma efetividade, que tenham melhor sobrevida e conforto para o paciente ${ }^{18,21}$

Os tipos de estudos utilizados nessa revisão sistemática foram ECR e ECC, pelo alto nível de evidência que apresentam, sendo assim, os resultados obtidos podem ser conclusivos. Porém, o único estudo incluído nos critérios estabelecidos compara dois métodos de confecção do aparelho mantedor de espaço arco lingual. $\mathrm{O}$ arco lingual é um aparelho fixo, de fácil construção, baixo custo e independe da colaboração do paciente para o uso. Pelo desenho que apresenta, previne o movimento mesial dos dentes posteriores e o movimento lingual dos dentes anteriores, diminuindo o apinhamento dental. É um mantedor não funcional, ou seja, não evita a extrusão dos antagonistas e, consequentemente, não reestabelece a função mastigatória ${ }^{24}$

$\mathrm{O}$ estudo incluído para esta revisão concluiu que o arco lingual, independente do calibre do fio utilizado para a confecção, foi efetivo para a manutenção do espaço, pois houve vestibularização dos incisivos inferiores e movimento distal dos molares permanentes por inclinação, porém, o arco confeccionado com fio de aço inoxidável $0,9 \mathrm{~mm}$ foi mais efetivo quando comparado com o mesmo aparelho confeccionado com fio $1,25 \mathrm{~mm}^{13}$. 
Essa diferença ocorreu devido às falhas que ocorreram com maior frequência no dispositivo de maior calibre, principalmente na cimentação, na quebra da banda e da solda. Todos os problemas foram resolvidos, salvo, a perda do aparelho, pois, mesmo havendo a falha de cimento, ocorreu sua remoção pelo paciente ou responsável, o que indicou falta de cooperação, e levou à exclusão do paciente do estudo ${ }^{13}$.

A dificuldade dessa revisão sistemática em selecionar artigos com delineamento que respondesse á questão de pesquisa incentiva o desenvolvimento de novos estudos com melhor delineamento. Nas buscas realizadas, foram encontradas pesquisas sobre os mantedores e suas falhas, principalmente comparando diferentes materiais para sua cimentação $0^{17,19}$ porém, uma lacuna com relação à efetividade e à comparação de diferentes tipos de mantedores foi constatada.

\section{Conclusão}

Tendo em vista os resultados obtidos nesta revisão sistemática, verifica-se que não é possível obter resultados conclusivos e concretos relativos à problemática proposta devido à carência de estudos existentes com esse delineamento. Sendo assim, faz-se necessário o desenvolvimento de novos estudos com delineamentos adequados para que a questão desta pesquisa possa ser respondida com um maior nível de evidência.

\section{Abstract}

Objective: To assess the current predictability of short and extra-short implants that are not splinted in the posterior mandible, and to discuss issues related to their classifications. Literature review: The rehabilitation of atrophic posterior areas presents major complexity for the planning and treatment with osseointegrated implants. Surgeries for height augmentation of alveolar ridge defects present no conclusive predictability by the literature; hence, implants with reduced length have been the target of several studies. The use of short implants decreases morbidity, surgical and treatment time, surgical complications, and the use of medication, in comparison to prior bone graft rehabilitations. Final considerations: According to the literature reviewed, the standardization of studies (human clinical trials) is required, aiming to establish success/survival rates fitting the particularities of short implants to provide definitions based on the predictability of these implants. Clinical trials with proper study design, providing higher results reliability, are essential for the clinical foundation of the dentist and for reducing bias.

Keywords: Dental implants. Alveolar bone loss. Survival rate.

\section{Referências}

1. Lino AP. Ortodontia preventiva básica. São Paulo: Artes Médicas; 1990.

2. Nogueira AJS. Perdas precoces de dentes decíduos e suas consequências para dentição futura - elaboração de propostas preventivas. Rev ABO Nac 1998; 6(4):228-33.

3. Graber TM. Ortodoncia: Teoria y Practica. 3. ed. México: Interamericana; 1974.

4. Corrêa MSNP. Mantenedores de espaço: que tipo e quando indicá-los. In: Todescan FF, Bottino MA. Atualização na clínica odontológica: a prática da clínica geral. São Paulo: Artes Médicas; 1996. p. 411-40.

5. Silva PRB. Mantenedores de espaço [Monografia de Especialização]. Bauru: Faculdade de Odontologia da Universidade de São Paulo; 1999.

6. Moyers RE. Ortodontia. 4. ed. Rio de Janeiro: Guanabara Koogan; 1991.

7. Van der Linden FPG. As consequências das perdas prematuras dos dentes decíduos. Ortodontia: Desenvolvimento da dentição. São Paulo: Quintessence; 1986.

8. Korytnicki D, Naspitz N, Faltin JRK. Consequências e tratamento das perdas precoces de dentes decíduos. Rev APCD 1994; 48(3):1323-9.

9. Pinkhan JR. Odontopediatria: da infância à adolescência. 2. ed. São Paulo: Artes Médicas; 1996.

10. Tomita NE. Relação entre hábitos bucais e má oclusão em pré-escolares. Rev Saude Publica 2000; 34(3):299-303.

11. Brothwell DJ. Guidelines on the use of space maintainers following premature loss of primary teeth. JAMA 1997; 63(10):753-66.

12. Nakata M, Wei Shy. Guia de Oclusão em Odontopediatria. 2. ed. São Paulo: Santos; 1995. 104 p. Colaboration. Oxford: Update Software; 2003.

13. Owais AI, Rousan ME, Badran SA, Abu Alhaija ES. Effectiveness of a lower lingual arch as a space holding device. Eur J Orthod 2010; 33(1):37-42.

14. Moher D, Liberati A, Tetzlaff J, Altman DG. The PRISMA Group. Preferred Reporting Items for Systematic Reviews and $M$ eta-Analyses: The PRISMA Statement. J Clin Epidemiol 2009; 62:1006-12.

15. Rajab LD. Clinical performance and survival of space maintainers: evaluation over a period of 5 years. ASDC J Dent Child 2002; 29(2):156-60.

16. Kirzioglu Z, Erturk MSO. Success of reinforced fiber material space maintainers. J Dent Child 2004; 71(2):158-62.

17. Moore TR, Kennedy DB. Bilateral Space maintainers: a 7-year retrospective study from private practice. Pediatr Dent 2006; 28(6):499-505.

18. Subramaniam P, Babu GKL, Sunny R. Glass fiber-reinforced composite resin as a space maintainer: A clinical study. J Indian Soc Pedod Prev Dent 2008; 26(Suppl 3): 98-103.

19. Sasa IS, Hasan AA, Qudeimat MA. Longevity of band and loop space maintainers using glass ionomer cement: a prospective study. Eur Arch Paediatr Dent 2009; 10(1):6-10.

20. Yeluri R, Munshi AK. Fiber reinforced composite loop space maintainer: An alternative to the conventional band and loop. Contemp Clin Dent 2012; 3(1):26-8.

21. Garg A, Samadi F, Jaiswal JN, Saha S. Metal to resin: a comparative evaluation of conventional band and loop space maintainer with the fiber reinforced composite resin space maintainer in children. J Indian Soc Pedod Prev Dent 2014; 32(2):111-6. 
22. Nisula KK, Hernesniemi R, Heiskanen M, Nisula KL, Varrela J. Orthodontic intervention in the early mixed dentition: A prospective, controlled study on the effects of the eruption guidance appliance. Am J Orthod Dentofacial Orthop 2008; 133(2):254-60.

23. Dincer M, Haydar S, Unsal B,Turk T. Space maintainer effects on intercanine arch width and length. J Clin Pediatr Dent 1996; 21(1):47-50.

24. Bonecker M, Guedes-Pinto AC, Fernandes FRC. Cárie dentária: In: Odontopediatria. 8. ed. São Paulo: Santos, 2010.

25. Mucha JN. Grampos e placas ortodônticas. Rio de Janeiro: Guanabara Koogan; 1997.

26. Simon T, Nwabueze I, Queis H, Stenger J. Space maintenance in the primary and mixed dentitions. J Mich Dent Assoc 2012; 94(1):38-40.

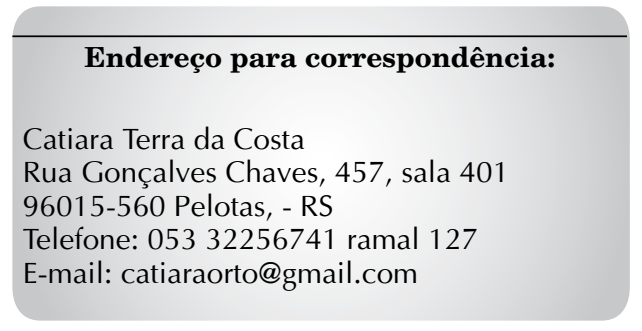

Recebido: 24/02/15. Aceito: 13/08/15. 